( $n=6$ healthy, 6 asthmatic): Compared to control, healthy ALI suppressed constitutive HLMC histamine release by $39 \pm 5 \%$ $(\mathrm{p}=0.01)$, but asthmatic ALI did not (mean $19 \pm 11 \%$ suppression, $p=0.07)$. There was a significant difference between healthy compared to asthmatic ALI $(p=0.01)$. Healthy and asthmatic ALI suppressed IgE-dependent histamine release by $55 \pm 4 \%, p=0.001$ and $48 \pm 1 \%, \mathrm{p}=0.001$, respectively.

Conclusions BEAS-2B and healthy airway epithelial cells suppress constitutive and IgE-dependent HLMC histamine secretion when separated by Transwell membranes. Asthmatic ALI cultures do not suppress constitutive HLMC histamine secretion, but do suppress IgE-dependent secretion. These results suggest that the normal regulation of this process is by a secreted, probably labile factor(s), which may be partially deficient in asthma. Isolation and manipulation of this factor may have interesting therapeutic potential.

\section{S34 EFFECTS OF THE CYCLIN-DEPENDENT KINASE INHIBITOR R-ROSCOVITINE ON EOSINOPHIL SURVIVAL AND CLEARANCE}

doi:10.1136/thx.2010.150912.34

${ }^{1} \mathrm{~N}$ Farahi, ${ }^{2} \mathrm{~L}$ Uller, ${ }^{1} \mathrm{~J} \mathrm{~K}$ Juss, ${ }^{1} \mathrm{~A} J$ Langton, ${ }^{1} \mathrm{~A} S \mathrm{~S}$ Cowburn, ${ }^{3} \mathrm{~A}$ Gibson, ${ }^{3} \mathrm{M}$ R Foster, ${ }^{3} \mathrm{~S} N$ Farrow, ${ }^{1} \mathrm{~A}$ Sobolewski, ${ }^{1} \mathrm{~A}$ M Condliffe, ${ }^{1} \mathrm{E}$ R Chilvers. ${ }^{1}$ University of Cambridge, Cambridge, UK; ${ }^{2}$ University of Lund, Lund, Sweden; ${ }^{3}$ GlaxoSmithKline, Stevenage, UK

Background Eosinophils are pro-inflammatory cells implicated in the pathogenesis of asthma and atopy. Apoptosis has been proposed as a potential mechanism underlying the resolution of eosinophilic inflammation and studies have indicated the ability of interventions that induce human eosinophil apoptosis to promote the resolution of eosinophilic inflammation. Recently, the cyclindependent kinase (CDK) inhibitor R-roscovitine was shown to enhance neutrophil apoptosis and promote the resolution of neutrophilic inflammation.

Aim The purpose of this study was to examine the expression of CDKs in human blood eosinophils, the effects of R-roscovitine on eosinophil survival and phagocytosis in vitro and determine whether R-roscovitine could influence eosinophilic lung inflammation in vivo. Methods Eosinophils were isolated from human peripheral blood and the effects of R-roscovitine on apoptosis, degranulation and phagocytic uptake examined in vitro. The effects of R-roscovitine on eosinophilic lung inflammation in vivo were also assessed using an ovalbumin mouse model.

Results Our data demonstrate that human eosinophils express five targets for R-roscovitine: CDK1, $-2,-5,-7$ and -9 . R-roscovitine induced eosinophil apoptosis in a time- and concentrationdependent manner but also accelerated transition to secondary necrosis as assessed by light and electron microscopy, flow cytometry and caspase activation. In addition, we report that the proapoptotic effect of R-roscovitine is associated with suppression of Mcl-1L expression and that the apoptotic eosinophils are phagocytosed by human monocyte derived macrophages. R-roscovitine also induced apoptosis in mouse eosinophils purified from the bonemarrow, spleen and peripheral blood. Despite this, R-roscovitine did not modulate the tissue and lumen eosinophilia characteristic of the ovalbumin mouse model of airway eosinophilia.

Conclusions These data demonstrate that R-roscovitine is capable of inducing rapid apoptosis and secondary necrosis in human eosinophils but does not affect the onset or resolution of eosinophilic airway inflammation in vivo.

\section{How should we be investigating suspected lung cancer?}

\section{S35 A RANDOMISED CONTROLLED TRIAL COMPARING COMBINED EBUS/EUS FOLLOWED BY SURGICAL STAGING VERSUS SURGICAL STAGING ALONE IN NON-SMALL CELL LUNG CANCER: THE ASTER STUDY}

doi:10.1136/thx.2010.150912.35

${ }^{1} \mathrm{R}$ C Rintoul, ${ }^{2} \mathrm{~K}$ G Tournoy, ${ }^{3} \mathrm{C}$ Dooms, ${ }^{3} \mathrm{P}$ De Leyn, ${ }^{4} \mathrm{~A}$ G Nicholson, ${ }^{2} \mathrm{E}$ Deschepper, ${ }^{5} \mathrm{O}$ M Dekkers, ${ }^{5} \mathrm{~K}$ F Rabe, ${ }^{2} \mathrm{~J}$ P van Meerbeeck, ${ }^{5} \mathrm{~J}$ T Annema. ${ }^{1}$ Papworth Hospital, Cambridge, UK; ${ }^{2}$ Ghent University Hospital, Ghent, Belgium; ${ }^{3}$ Leuven University Hospital, Leuven, Belgium; ${ }^{4}$ Royal Brompton Hospital, London, UK; ${ }^{5}$ Leiden University Medical Centre, Leiden, The Netherlands

Background For many years the standard approach to staging of the mediastinum in non-small cell lung cancer (NSCLC) has been surgical using cervical mediastinoscopy, left anterior mediastinotomy or video assisted thoracoscopic surgery (VATS). More recently endobronchial ultrasound (EBUS) and endoscopic ultrasound (EUS) have been reported. We conducted a randomised phase III trial to compare surgical staging versus endoscopic staging. The primary endpoint was detection of mediastinal nodal metastasis (N2/3); secondary endpoints were complication and futile thoracotomy rates.

Methods Consecutive patients with potentially resectable (suspected) NSCLC in whom invasive mediastinal staging was indicated based on CT or PET-CT findings were randomly assigned to either Arm A, surgical staging or Arm B, combined EBUS/EUS (followed by surgical staging if endoscopic findings were negative for malignancy). Surgical staging involved mediastinoscopy and/or mediastinotomy and/or VATS. Subsequently, in the absence of mediastinal disease, thoracotomy with systematic lymph node sampling was performed. 240 pts were required to show a $20 \%$ increase in sensitivity (power $80 \%$ and $\alpha=0.05$ ) to detect mediastinal nodal disease with a prevalence of $50 \%$.

Results 118 patients were randomised to Arm A and 123 to Arm B. The sensitivity for detection of mediastinal metastases by surgical staging in Arm A was 80\% (95\% CI, 68 to 89) vs 94\% (95\% CI, 85 to 98) for endoscopic ( \pm surgical) staging in Arm B ( $p=0.04)$. Nodal metastases were found in 41 (35\%) of surgically staged patients in Arm $\mathrm{A}$ and 62 patients (50\%) (56 by EBUS/EUS +6 by subsequent surgical staging) in Arm B ( $p=0.019)$. Overall, the prevalence of mediastinal disease in each arm was similar $(p=0.24)$. Thoracotomy was considered futile in $21(18 \%)$ in those staged in Arm A vs 8 patients $(7 \%)$ in Arm B ( $p=0.009)$. Complication rate was similar in both arms ( 6 vs 7 patients, $p=0.8$ ); however, 12 of 13 complications were due to surgical staging procedures.

Conclusions Mediastinal staging for NSCLC should commence with combined EBUS/EUS (followed by surgical staging if endoscopic findings are negative for malignancy) as this improves the detection of nodal metastases and reduces futile thoracotomies compared to surgical staging alone.

\section{S36 CHECK NOVEL IN VIVO REAL TIME IMAGING OF THE BRONCHIAL MUCOSA USING AN ENDO-CYTOSCOPY}

doi:10.1136/thx.2010.150912.36

\begin{abstract}
${ }^{1,3}{ }^{K}$ Shibuya, ${ }^{1} \mathrm{~N}$ Okada, ${ }^{1} \mathrm{H}$ Kohno, ${ }^{1} \mathrm{~N}$ Iwai, ${ }^{2} \mathrm{M}$ Noro, ${ }^{2} \mathrm{~B}$ Akikusa, ${ }^{3} \mathrm{H}$ Hoshino, ${ }^{3}$ Y Yoshino. ${ }^{1}$ Department of Chest Surgery, Matsudo City Hospital, 4005 Kamihongo, Matsudo, 271-8511, Japan; 'Department of Pathology, Matsudo City Hospital, 4005 Kamihongo, Matsudo, 271-8511, Japan; ${ }^{3}$ Department of General Thoracic Surgery, Graduate School of Medicine, Chiba University, 1-8-1 Inohana, Chuo-ku, Chiba 260-8670, Japan
\end{abstract}

Objectives We investigated the capabilities of an Endo-Cytoscopy system (ECS) that enables microscopic imaging of the 
tracheobronchial tree during bronchoscopy, including normal bronchial epithelium, dysplastic mucosa and hilar lung cancer.

Methods The newly developed integrated-type ECS for the bronchoscope has a built-in two imaging system with a conventional mode and a high-power endocytoscopic mode. ECS has a high magnification of $570 \times$. Thirty-seven patients including 9 hilar lung cancer, 6 abnormal sputum cytology, 19 squamous dysplasia, and 3 after photodynamic therapy were entered into the study and underwent white light, narrow band imaging and autofluorescence imaging bronchoscopy. Both the abnormal area of interest and surrounding normal bronchial mucosa were stained with $0.5 \%$ methylene blue and examined with ECS. Histological examinations with haematoxylin and eosin stain were performed using the biopsied specimens. The ECS imaging was analysed and correlated with the corresponding histological examination.

Results ECS imaging could distinguish between different types of bronchial epithelium including normal bronchial mucosa, squamous dysplasia, and hilar lung cancer. Squamous dysplasia and hilar lung cancer were predictive with sensitivity of $85.7 \%(12 / 14)$ and $90.9 \%$ $(10 / 11)$ and specificity of $100 \%$ (12/12), respectively. These ECS images corresponded well conventional histology.

Conclusion ECS was useful for the discrimination between normal bronchial epithelial cells and dysplastic cells or malignant cells during bronchoscopy in real time. This novel technology has an excellent potential to provide in vivo diagnosis during bronchoscopic examinations.

\section{S37 COMPARISON OF DYNAMIC CONTRAST ENHANCED MRI (DCE-MRI) PARAMETERS WITH INTEGRATED PET-CT AND SERUM MESOTHELIN IN THE BASELINE ASSESSMENT OF MALIGNANT PLEURAL MESOTHELIOMA}

doi:10.1136/thx.2010.150912.37

${ }^{1} \mathrm{C}$ Hooper, ${ }^{2} \mathrm{D}$ Hall, ${ }^{3} \mathrm{P}$ Virgo, ${ }^{4} \mathrm{P}$ White, ${ }^{3} \mathrm{M}$ Darby, ${ }^{5} \mathrm{~T}$ Hall, ${ }^{6} \mathrm{~J}$ Braybrooke, ${ }^{2} \mathrm{~J}$ Searle, ${ }^{2}$ L Lyburn, ${ }^{1} \mathrm{~N}$ Maskell. ${ }^{1}$ Academic Respiratory Unit, Department of Clinical Sciences, University of Bristol, Southmead Hospital, Bristol, UK; ${ }^{2}$ Cobalt Cheltenham Imaging Centre, Cheltenham, UK; ${ }^{3}$ Southmead Hospital, North Bristol NHS Trust, Bristol, UK; ${ }^{4}$ University of the West of England, Bristol, UK; ${ }^{5}$ Royal United Hospital Bath, Bath, UK; ${ }^{6}$ Bristol Oncology Centre, UBHT, Bristol, UK

Integrated PET-CT scans and serum mesothelin measurement have shown early promise in predicting prognosis and evaluating treatment response in malignant pleural mesothelioma (MPM) but may be less reliable with sarcomatoid histology or prior talc pleurodesis. Dynamic Contrast Enhanced-MRI (DCE-MRI) with pharmacokinetic analysis is a novel metabolic imaging modality providing a measure of tumour blood flow and angiogenesis. We prospectively examined the relationship between pharmacokinetic parameters on DCE-MRI with PET-CT, serum mesothelin and histological subtype in MPM patients at diagnosis.

Method 30 pre-treatment patients with a histologically proven MPM underwent DCE-MRI and integrated PET-CT and serum mesothelin assay (MESOMARK) at a single visit. SUVmax and total glycolytic volume (TGV) were reported from PET-CT scans with TGV calculated using MIM software version 4.2.2 (MIMvista corp.). Gadolinium washout rate (GWR) on DCE-MRI was defined at a region of interest from a straight line fit to the kinetic curve data (CAD software-ViewForum R6.3 V1L3, Philips Medical Systems) between peak enhancement in the first $2 \mathrm{~min}$ and the last data point.

Results 70\% (21/30) epithelioid and 30\% (9/30) sarcomatoid histology. $43 \%(13 / 30)$ had undergone prior talc pleurodesis. Histology did not statistically significantly affect SUVmax, TGV or GWR. Serum mesothelin was significantly greater in the epithelioid group $(3.2 \mathrm{nM} / 1(2.0,6.3)$ vs $0.6 \mathrm{nM} / 1(0.5,0.8) \mathrm{p}<0.001)$. There was no significant difference in mesothelin, SUVmax, TGV or GWR between talc pleurodesed and non-pleurodesed patients in the whole group, but in the epithelioid sub-group there was a trend to significantly higher TGV with talc pleurodesis (talc: $2799(1931,11257)$ no talc: $955.5(146.8,2354) \mathrm{p}=0.053)$ that was not observed with GWR $(p=0.4179)$. While SUVmax strongly correlated to TGV $(r=0.725$, $\mathrm{p}<0.001$ ), there was no correlation between GWR and TGV $(r=0.203, p=0.282)$ or between mesothelin levels and any of the imaging values.

Conclusion Metabolic imaging has been proposed as an important component of the assessment and management of patients with malignant pleural mesothelioma. Gadolinium washout rate on DCE-MRI may be less sensitive to talc pleurodesis than PET-CT parameters and MRI is a cheaper, more readily available modality that involves shorter patient appointment times, warranting further study in MPM prognostic evaluation and treatment response monitoring.

\section{S38 FOURIER TRANSFORM INFRA-RED (FTIR) SPECTROSCOPY ON SPUTUM FROM LUNG CANCER PATIENTS, HEALTHY CONTROLS AND A HIGH-RISK COHORT}

doi:10.1136/thx.2010.150912.38

${ }^{1} \mathrm{R}$ Ghosal, ${ }^{1} \mathrm{~K}$ E Lewis, ${ }^{2} \mathrm{P}$ Kloer, ${ }^{1} \mathrm{~S}$ Bayliss, ${ }^{3} \mathrm{~L}$ Mur, ${ }^{1} \mathrm{P} \mathrm{D}$ Lewis. ${ }^{1}$ School of Medicine, Swansea University, Swansea, UK; ${ }^{2}$ Prince Philip Hospital, Llanelli, UK; ${ }^{3}$ Institute of Biological Sciences, Aberystwyth University, Aberystwyth, UK

Introduction New cheap and high throughput technologies may allow earlier diagnosis and cost-effective screening programmes for lung cancer (LC). We have shown that sputum is a feasible biofluid for FTIR spectroscopy analysis ${ }^{1}$ and now further evaluate FTIR in diagnosing LC.

Methods Sputum was taken from three groups: a) 54 patients (mean age $66.6 \pm 8.7$ years) with a histological diagnosis of LC (39 NSCLC, 9 small cell, 1 carcinoid, 5 clinical diagnosis). b) 24 patients (mean age 65.1 \pm 13.6 years) having bronchoscopy for possible LC

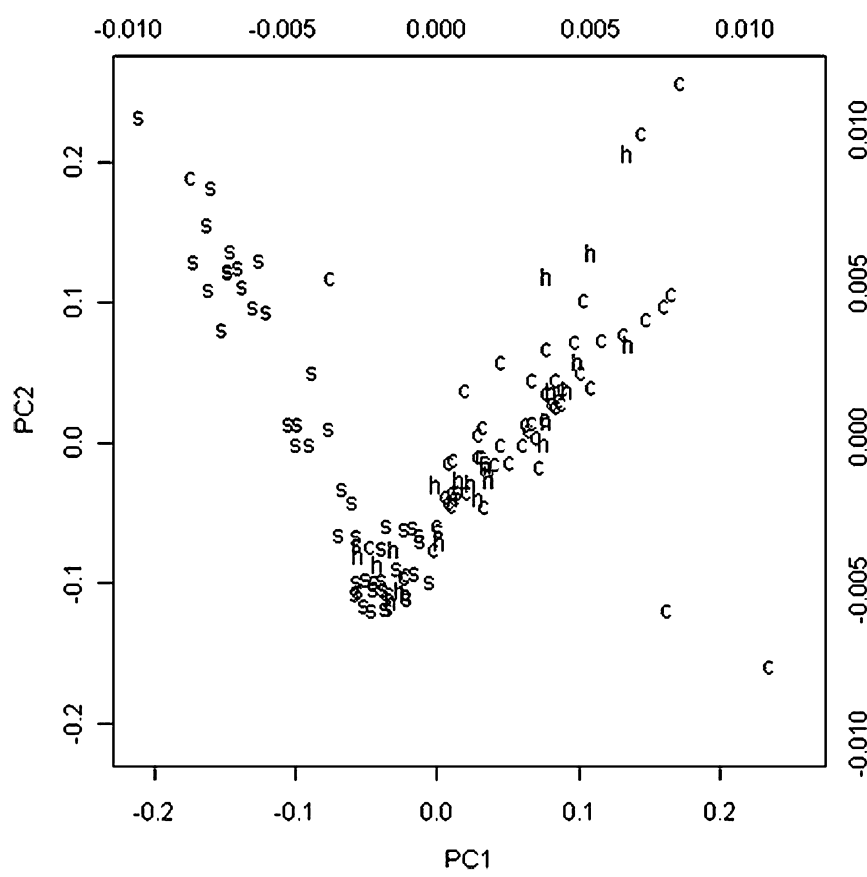

Abstract S38 Figure 1 Principal component analysis of cancer (c), healthy control (s) and high-risk (h) spectra. 